\title{
EMC/EMI Analysis in Wireless Communication Networks
}

\author{
S. Loyka \\ Department of Electrical Engineering \\ Ecole de Technologie Superieure \\ 1100, Notre-Dame St. West \\ Montreal (Quebec), H3C 1K3, CANADA \\ Email: sergey.loyka@ieee.org
}

\begin{abstract}
Main challenges in EMC/EMI modeling and simulation for modern and future wireless communication networks are addressed in this paper. It is emphasized that there exists a big difference between simulating analog and digital systems. A computationally-efficient approach for EMC/EMI analysis of wireless networks by behavioural-level numerical simulation is proposed.
\end{abstract}

\section{INTRODUCTION}

Wireless communication systems experience today unprecedented growth. The number of systems increases almost exponentially. In view of very limited spectrum available and large concentration of system over limited space (for example, in dense urban or indoor environments), the potential for mutual interference is tremendous. There are three potential wireless "candidates" for large EMC/EMI problems in the near future.

First, the implementation of advanced smart antennas in $3 \mathrm{G}$ wireless systems, which allows to improve their performance substantially [1, 2], may cause additional EMC/EMI problems because a typical branch of antenna array, which is the main structure for smart antennas, contains active (nonlinear) stages. These stages can produce a considerable amount of RF EMI, especially in heavily-overcrowded spectrum [3, 4]. Secondly, the large-scale introduction of broadband wireless systems [5, 6], which is foreseen for near future, will make these problems even more important and more difficult to solve since any bandwidth expansion increases the risk of getting interference problems. Thirdly, Bell Labs Layered Space-Time (BLAST) communication architecture, also known as MIMO (multiple-input multiple-output), is likely to be implemented in the near future due to its unprecedented spectral efficiency [7, 8]. This architecture uses multiple transmit and receive branches to launch and detect independent bit streams over the same wireless channel. Thus, its Tx and Rx branches operate at the same frequency, which can be a source of many EMC/EMI problems, especially in a complex electromagnetic environment [3]. The complexity of $\mathrm{EMC} / \mathrm{EMI}$ analysis in a future wireless network is very large due to the large number of systems in the network and due to the complexity of systems (many Tx/Rx branches, complex structure of every branch) and of the electromagnetic environment.
Generally speaking, the performance of wireless communication networks can be severe degraded by mutual interference between various radio systems sharing the same space and the same frequency band and, thus, must be carefully taken into account in the design and implementation (deployment) stages. The techniques proposed in the past [911] are largely based on empirical or semi-empirical models developed for analog systems and are, in general, not accurate for modern digital systems.

In this paper, we discuss main challenges in EMC/EMI modeling and simulation for wireless communication networks, main differences between EMC/EMI analyses for analog and digital systems and propose a computationallyefficient approach for system-level simulation of wireless systems at the physical layer (i.e., taking into account almost all the hardware imperfections) which can be efficiently combined with the existing methods of simulation at the signal processing level providing in this way a technique for the simulation of the entire communication link, starting for the source of information and up to the destination point. The computational efficiency of this approach is expected to be high enough to allow the simulation of real-world systems under real-world conditions in reasonable time and using reasonable computational resources.

\section{EMI in ANALOG AND Digital WIRELESS SySTEMS}

The mutual interference between wireless systems is presently taken into account mainly during the frequency planning stage and in an oversimplified way, i.e. no comprehensive Tx and $\mathrm{Rx}$ models are employed, only few basic parameters (carrier frequency, bandwidth, Tx power, Rx sensitivity and selectivity etc.) are accounted for. The main reason for this is that there is no accurate and computationally-efficient technique available for more accurate and comprehensive analysis. Thus, even carefully made frequency planning does not guarantee the absence of EMI in any scenario.

In general, all the interference effects may be divided into two large groups: (1) linear effect and (2) nonlinear effect. Modeling and simulation of linear effects in communication systems do not present a serious problem and can be carried out in a conventional way [11]. On the contrary, accurate modeling and simulation of nonlinear effects in modern communication systems is a big challenge [12]. Thus, we 
concentrate our discussion on modeling and simulation of nonlinear effects.

Some possible kinds of nonlinear interference and distortions for wireless communication systems are the following $[9,10]$ :

1. Spurious radiation of transmitters: harmonics and subharmonics, intermodulation products (IMP), noise etc. Note that the intermodulation radiation can be caused by an external signal (from a nearby transmitter) as well as by several carriers (in CDMA systems, for example) in the transmitter's power amplifier.

2. Spurious responses of receivers: adjacent, image and intermediate frequency (IF) channels.

3. Nonlinear behavior of receivers may also cause the system performance degradation (desensitization, IMPs, local oscillator noise and harmonics' conversion etc.).

Analytical and semi-empirical methods were extensively used in the past for the analysis of these effects [9, 10]. Those methods were quite simple and allowed one to get some insight into the system operation. However, the accuracy of those methods is rather poor. Besides, the analysis of complex systems (present-day digital wireless systems) is rather difficult if not possible at all to do by the old methods.

Digital communication systems present additional challenge for the EMC/EMI analysis because computational resources required for such an analysis is much higher for digital systems than for analog ones [12]. For example, for a RF power amplifier employed in an analog system, IMP levels present its important performance parameter (among others). The traditional two-signal simulation is sufficient to predict this performance parameter. On the contrary, if this amplifier is employed in a digital system, other performance measures are used - adjacent channel power ratio (ACPR), error vector magnitude (EVM) etc., because the traditional analog parameters (i.e., IMPs, IPs etc.) do not represent adequately the digital system performance [12]. In order to predict these new parameters, multi-tone simulation should be used (typically, with few thousand or even tens of thousand tones), which is much more computationally-expensive. A similar set of new parameters should be employed to predict adequately the performance of digital wireless receivers.

The considerations above suggest that the only likely candidate for the EMC/EMI analysis of present-day and future wireless systems is a numerical simulation approach. A system-level analysis, which is required in our case, cannot be done at the circuit-level due to high complexity of the wireless system and of the digitally modulated signals. Besides, the circuit-level simulation would require too much characterization data. Thus, behavioral-level techniques (also known as "black-box" techniques) should be used for such a problem $[3,13,14]$. We further discuss three such techniques.

\section{QUADRATURE Modeling TeCHNIQUE}

The quadrature modeling technique is the most popular tool for behavioral-level nonlinear modeling and simulation of the active stages of communication circuits and systems $[11,15]$. This technique was introduced in early 1970s for nonlinear modeling and simulation of traveling wave tube amplifiers used in satellite communications. The main idea of the quadrature modeling technique is the use of a complex envelope instead of real narrowband signals:

$$
\begin{aligned}
x(t)= & A(t) \cos \left(\omega_{0} t+\varphi(t)\right)= \\
& \operatorname{Re}\left\{A(t) \cdot \exp \left[j \cdot\left(\omega_{0} t+\varphi(t)\right)\right]\right\}
\end{aligned}
$$

where $A(t)$ and $\varphi(t)$ - are amplitude and phase that vary slowly with respect to carrier (amplitude and phase modulation), $\omega_{0}-$ is the carrier frequency. Its complex envelope is

$$
\overline{A(t)}=A(t) \cdot \exp [j \varphi(t))]
$$

So, there is not any carrier information in the complex envelope, only modulation information (only the first harmonic zone is taken into account). It's very important from the viewpoint of computational efficiency, but it also limits the technique capabilities - only narrowband analysis is possible because the frequency response is assumed to be flat over the simulation bandwidth. The output signal of a bandpass nonlinear stage is

$$
\begin{aligned}
& y(t)=K\left(A_{\text {in }}(t)\right) \cdot A_{\text {in }}(t) \times \\
& \cos \left(\omega_{0} t+\varphi_{\text {in }}(t)+\Phi\left(A_{\text {in }}(t)\right)\right)
\end{aligned}
$$

where $A_{\text {in }}$ and $\varphi_{\text {in }}$ are the input signal amplitude and phase. A nonlinear stage is characterized by its envelope amplitude and phase transfer factors:

$$
K\left(A_{\text {in }}\right)=\frac{A_{\text {out }}}{A_{\text {in }}}, \quad \Phi\left(A_{\text {in }}\right)=\varphi_{\text {out }}-\varphi_{\text {in }}
$$

$K\left(A_{\text {in }}\right)$ represents envelope amplitude-to-amplitude (AM-AM) nonlinearity, and $\Phi\left(A_{\text {in }}\right)$ represents envelope amplitude-tophase (AM-PM) nonlinearity. Note that both factors depend on the input signal amplitude, not on instantaneous value of the signal. It's due to the bandpass representation of signals and system stages (actually, lowpass equivalents of both are used). Thus, equation (4) constitutes the envelope nonlinearity.

In the quadrature modeling technique, in-phase and quadrature envelope transfer factors are used

$$
\begin{aligned}
& K_{I}\left(A_{\text {in }}\right)=K\left(A_{\text {in }}\right) \cos \Phi\left(A_{\text {in }}\right) \\
& K_{Q}\left(A_{\text {in }}\right)=K\left(A_{\text {in }}\right) \sin \Phi\left(A_{\text {in }}\right)
\end{aligned}
$$

and output lowpass signal is expressed as

$$
Y(t)=K_{I}\left(A_{\text {in }}\right) X_{I}(t)-K_{Q}\left(A_{\text {in }}\right) X_{Q}(t)
$$

Two independent channels (in-phase (I) and quadrature (Q)) are used for the simulation. In this way this technique takes into account both the AM-AM and AM-PM nonlinearities. This nonlinear model is sometimes called a memoryless nonlinearity in the sense that the transfer factors $K_{I}$ and $K_{Q}$ 
depend on the input signal amplitude $A_{\text {in }}$ at the same instant only. [13].

At the present time, this technique is mainly used for the simulation of solid-state power amplifiers. It has many advantages: it allows one to simulate the power amplifier with a digitally-modulated input signal using a PC in reasonable time and, consequently, to predict ACPR, power spectral regrowth (PSR) and EVM. The technique can also predict IMPs and gain compression/expansion. The permissible number of input tones and the analysis dynamic range are quite large. However, the main drawback of the quadrature modeling technique is that it is a narrowband one, so it can not take into account frequency response, to predict harmonics of the carrier frequency and even-order nonlinear products, or to model the bias decoupling network effect [13]. This effect limits the analysis accuracy even for narrowband signals and systems. The quadrature modeling technique also uses an explicit representation of the modulated signal, so multiplecarrier signals can not be simulated in a direct way. Thus, some improvements are desirable.

\section{DISCRETE TECHNIQUE}

The discrete technique was introduced in 1980s for the nonlinear simulation of a $\mathrm{RF} /$ microwave receiving path taking into account nonlinear interference and distortions [16, 17]. It also accounts for the spurious receiver channels (adjacent, image, local oscillator noise etc.), IMPs and harmonics, gain compression/expansion etc. The main application of this technique was to the EMC/EMI analysis in a group of $\mathrm{RF} /$ microwave systems. An important advantage of the discrete technique is that instantaneous values of the signals are used during the analysis, not the complex envelope.

The basis of the discrete technique is a representation of the system block diagram as linear filters (matching networks) and memoryless nonlinear elements (active elements) connected in series (or in parallel, or both) $[3,13,16,17]$. Input and output filters model input and output matching networks correspondingly. This representation reflects characteristic peculiarities inherent to the structure of typical RF amplifying and converting stages. Signal passage through a linear filter is simulated in the frequency domain using the complex transfer factor of the filter,

$$
S_{\text {out }}(f)=S_{\text {in }}(f) \cdot K(f),
$$

where $S_{\text {out }}(f)$ - is the output signal spectrum, $S_{\text {in }}(f)$ - is the input signal spectrum, $K(f)$ - is the filter complex transfer factor, and $f$-is frequency. An appropriate sampling technique is required in order to sample the spectrum. Signal passage through a nonlinear memoryless element is simulated in the time domain using the instantaneous transfer function of the element,

$$
u_{\text {out }}(t)=F\left[u_{\text {in }}(t)\right]
$$

where $u_{\text {out }}(t)$ - is instantaneous value of the output signal at time instant $t, u_{\text {in }}(t)$ - is the same for input signal, $F$ - is an instantaneous transfer function of the nonlinear element. This function can be calculated using the measured or circuit-level simulated AM-AM characteristic [13].

Thus, the simulation is made over a wide frequency range. The technique allows one to predict harmonics and even-order nonlinear products, to take into account frequency response and to analyze multi-carrier systems. Both these techniques (the quadrature modeling technique and the discrete technique) are very computationally efficient as compared to circuit-level techniques (harmonic balance (HB) technique or SPICE, for example), and still have a circuit-level accuracy in many cases. However, the discrete technique does not take into account AM-PM nonlinearity that limits substantially the analysis accuracy.

\section{INSTANTANEOUS QUADRATURE TECHNIQUE}

The main idea behind the instantaneous quadrature technique to combine the quadrature modeling technique and the discrete technique in order to build a simulation technique which can model the circuit or system behavior over wide frequency and dynamic ranges taking into account both AM-AM and AMPM conversions [3, 13, 14]. In order to model signals and systems over a wide frequency range, the instantaneous values of the signals must be used, not the complex envelope. In order to model the AM-PM conversion, the quadrature modeling structure should be used for the nonlinear element modeling. Thus, the modeling process consists of the following items:

1. Linear filters are modeled in the frequency domain (the same as for the discrete technique).

2. Nonlinear elements are modeled in the time domain using the quadrature structure, but the instantaneous signal values are used, not the complex envelope.

3. The transform from the frequency (time) domain to the time (frequency) domain is made by IFFT (FFT) (very computationally efficient).

4. The Hilbert transform [3] is used to calculate in-phase and quadrature components,

$$
x_{Q}(t)=\hat{x}(t)=\int_{-\infty}^{\infty} \frac{x(\tau)}{t-\tau} d \tau, x_{I}(t)=x(t),
$$

where $x_{I}(t)$ and $x_{Q}(t)$ - are instantaneous in-phase and quadrature components of the signal, $\hat{x}(t)$ is the Hilbertconjugate signal of $x(t)$. In fact, we use the Hilbert Transform in the frequency domain to calculate the quadrature components because it does not require numerical integration and, thus, is much more computationally efficient:

$$
\begin{array}{r}
x_{Q}(t)=\operatorname{IFFT}(-j \cdot S(\omega)) \text { for } \omega \geq 0, \\
S(\omega)=F F T(x(t))
\end{array}
$$

where $S(\omega)-$ is the signal's spectrum. The signal itself is the in-phase component, and the Hilbert's conjugate signal is the quadrature component. 


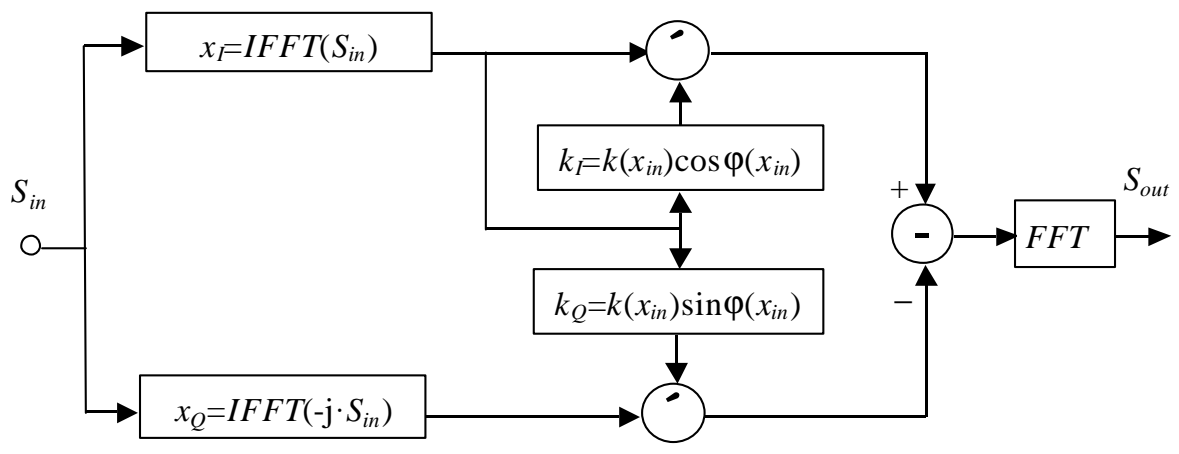

Figure 1. Modeling broadband nonlinear element by the instanteneous quadrature technique.

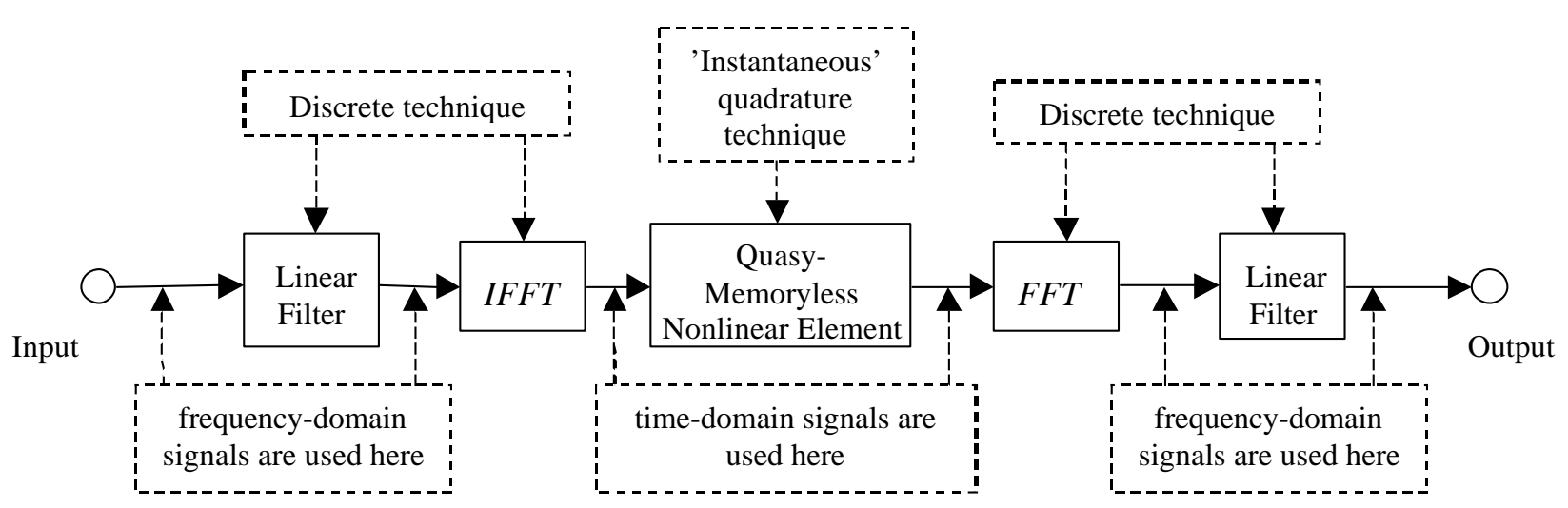

Figure 2. Simulating a single-stage radio amplifier by the instantaneous quadrature technique.

5. A system of two integral equations is used in order to convert the envelope transfer function into the instantaneous ones:

$$
\begin{aligned}
& \frac{4}{\pi} \int_{0}^{1} k_{I}\left(A_{i n} t\right) \frac{t^{2} d t}{\sqrt{1-t^{2}}}=K\left(A_{i n}\right) \cos \Phi\left(A_{i n}\right) \\
& \frac{4}{\pi} \int_{0}^{1} k_{Q}\left(A_{i n} t\right) \sqrt{1-t^{2}} d t=K\left(A_{i n}\right) \sin \Phi\left(A_{i n}\right)
\end{aligned}
$$

where $k_{I}$ and $k_{Q}$ - are the instantaneous in-phase and quadrature transfer factors. Note also that using (11) only the even parts of the transfer factors can be calculated. In order to find the odd parts, some additional characteristics should be used (for instance, the second harmonic transfer factor). As a rule, the amplitude transfer characteristics can be measured or simulated using a circuit-level simulator, thus we need to solve equations (11) for $k_{I}$ and $k_{Q}$. This can be done using the method of moments. If we use piecewise constant basis functions and a point matching technique, the matrices of these equations appear to be upper triangular ones, so the systems of linear equations can be solved analytically (this semi-analytical approach speeds up computations substantially).
Fig. 1 gives an illustration of the nonlinear element modelling. It should be noted that the transfer function of the quadrature channel is not a usual transfer function in a conventional sense: it depends not only the input of the quadrature nonlinearity $\left(x_{Q}\right)$ but also on the input of the entire structure $\left(x_{i n}\right)$ i.e., in fact, on the input of the in-phase nonlinearity $\left(x_{I}\right)$ [13]. Consequently, we cannot use the usual methods of the transformation of the envelope transfer function into the instantaneous one. At the same time, the transfer function of the in-phase channel is a usual transfer function and those methods can be applied in this case.

If one needs to simulate even-order nonlinear products, then odd parts of the instantaneous transfer factors must be determined. It can be done using the second-order envelope characteristics (second harmonic zone AM-AM and AM-PM functions or second-order IMP at the output for the two-tone input):

$$
\begin{aligned}
& \frac{4}{\pi} \int_{0}^{1} k_{I}\left(A_{i n} t\right) \frac{t\left(2 t^{2}-1\right)}{\sqrt{1-t^{2}}} d t=K_{2}\left(A_{\text {in }}\right) \cos \left(\Phi_{2}\left(A_{\text {in }}\right)\right) \\
& \frac{8}{\pi} \int_{0}^{1} k_{Q}\left(A_{i n} t\right) t \sqrt{1-t^{2}} d t=K_{2}\left(A_{\text {in }}\right) \sin \left(\Phi_{2}\left(A_{\text {in }}\right)\right)
\end{aligned}
$$




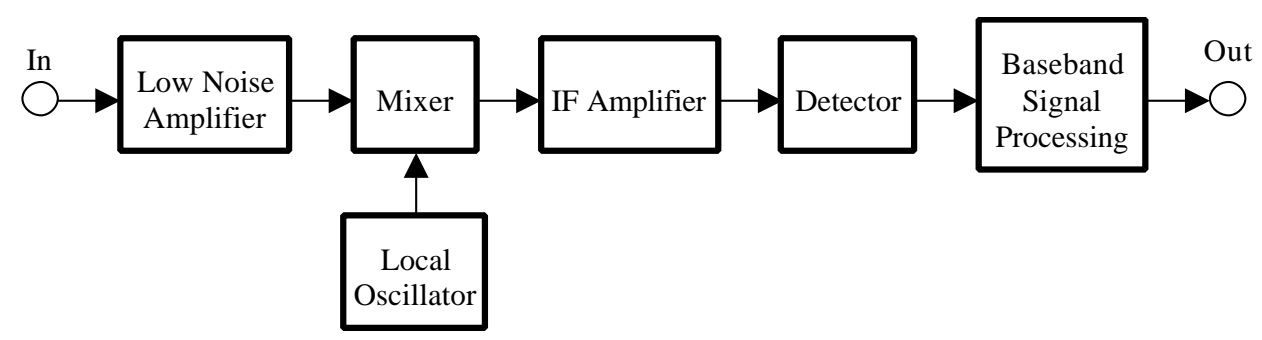

Figure 3. Simplified block diagram of a wireless receiver; digital signal processing is after detector.

where $K_{2}\left(A_{\text {in }}\right)$ is the second-order envelope transfer factor and $\Phi_{2}\left(A_{i n}\right)$ is the second-order AM-PM characteristic. We can solve the integral equations (12) using the traditional method of moments approach. But, as practical experience shows, it requires even more sample points than for the even parts. Thus, it is much efficient to use the semi-analytical approach proposed above.

\section{APPlying InStantaneous QUadrature TechNique to WiRELESS SYSTEMS}

Let us now consider the simulation of typical wireless subsystems/systems using the instantaneous quadrature technique. Fig. 2 illustrates the simulation of a single stage RF amplifier (either power or low noise). The input and output filters model the matching networks, and the quasymemoryless nonlinear elements model the amplifier nonlinearity (both AM-AM and AM-PM). Using this approach, we may simulate other RF and IF stages (like mixers and IF amplifiers) and even detectors [18] in a computationally-efficient way. Further, using the black-box approach, we may simulate the entire link and network operation under real-world conditions and signals.

The simulation of a wireless transmitter using the instantaneous quadrature technique is quite straight-forward: we do it in the same way as for the quadrature modeling technique. The power amplifier is considered to be the main source of interference and distortions in this case (however, other stages can also be simulated).

Figure 3 shows the simplified block diagram of a wireless receiver (digital signal processing is after detector), in a form suitable for the EMC/EMI analysis (filters are included in the corresponding blocks). Automatic gain control, automatic frequency control and synchronization loops are not shown because they are not easy to simulate using the techniques above in its present form. This is an important topic for future research. The simulation of a wireless receiver is not so simple because many its components can contribute to the overall interference and distortions. Thus, we consider (i.e. simulate) the receiver block diagram step by step: low-noise amplifier, local oscillator plus mixer, IF filter and IF amplifier, detector and baseband signal processing part. It should be noted that (i) frequency response is taken into account in every stage, and (ii) detector simulation is possible using the technique proposed that enables us to go further to the simulation of baseband signal processing. DSP can be simulated using either the traditional approach [11] or the instantaneous quadrature technique. Thus, the instantaneous quadrature technique can be used for the EMC/EMI simulation of the entire wireless communication link over wide frequency and dynamic ranges.

\section{TeChNiQue VALIDATION}

In order to validate the instantaneous quadrature technique, extensive harmonic-balance simulations as well as measurements of microwave solid-state amplifiers have been carried out [13]. Fig. 4 shows IMPs simulated by the instantaneous quadrature technique (solid line) and by the harmonic balance technique (squares). One can note quite a good agreement between behavioral-level and harmonic balance (circuit level) simulations. In general, the discrepancy is about few $\mathrm{dBs}$ except for some special areas, which should be further investigated. Fig.5 shows harmonics simulated by the instantaneous quadrature technique (solid line) and measured (squares). Behavioral-level simulation and measurements agree quite well in this case as well. The discrepancy is rather large for several special areas, which should be further investigated. Note also that the analysis is made over wide dynamic range (130-180 dB) and wide frequency range (harmonics!). Thus, these results seem to be very satisfactory taking into account that the problem is a nonlinear one. Accuracy of even-order nonlinear products prediction is slightly worse but still satisfactory for practical purposes.

\section{CONCLUSION}

In this paper, the main challenges in EMC/EMI analysis for present and future wireless communication networks have been discussed. It was emphasized that there was a big difference between modelling and simulation of digital and analog wireless systems. Behavioral-level numerical simulation seems to be a suitable tool for the EMC/EMI analysis of digital wireless systems and networks. Three behavioural-level techniques have been discussed with emphasis on the instantaneous quadrature technique as the most advanced one. This techniques combines the advantages of both the quadrature modeling technique and the discrete technique and, consequently, gives one possibility to simulate 


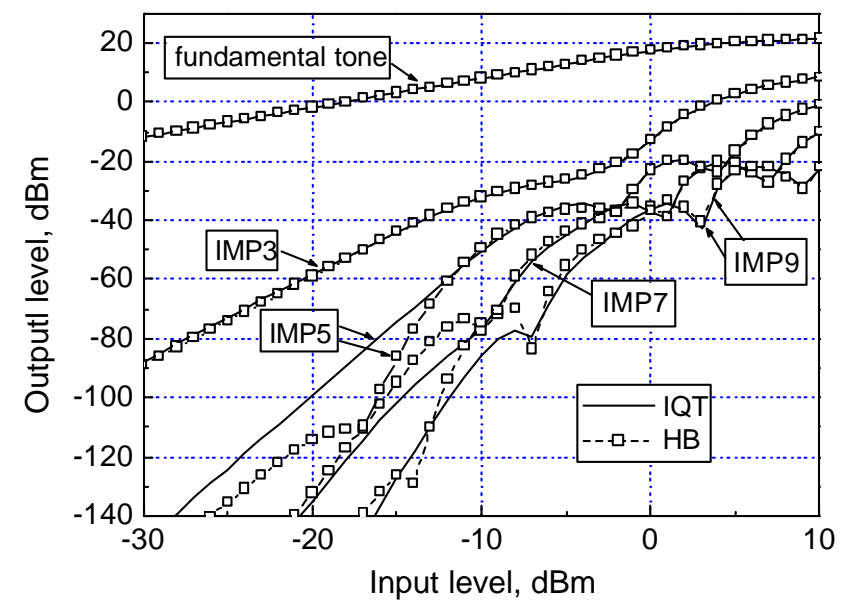

Figure 4. Fundamental and $3^{\text {rd }}$ to $9^{\text {th }}$ order IMPs at the microwave amplifier output (solid line - instantaneous quadrature technique, squares - harmonic balance technique).

the circuit/system nonlinear performance over wide frequency and dynamic ranges. This technique should be used on the frequency planning phase in order to avoid possible system performance degradation on the implementation phase and to ensure reliable and high-quality communication.

\section{REFERENCES}

[1] J.H. Winters, Smart Antennas for Wireless Systems, IEEE Personal Communications, Feb. 1998, pp. 23-27.

[2] K. Sheikh et al, Smart Antennas for Broadband Wireless Access Networks, IEEE Communications Magazine, vol. 37, N. 11, Nov. 1999, pp. 100-105.

[3] S.L. Loyka, "The Influence of Electromagnetic Environment on Operation of Active Array Antennas: Analysis and Simulation Techniques," IEEE AP Magazine, Dec. 1999, vol. 41, N 6, pp. 23-39.

[4] M. Wennstrom, Smart Antenna Implementation Issues for Wireless Communications, M.S. thesis, Uppsala University, Uppsala, Sweden, 1999.

[5] M. Shafi et al, Wireless Communications in the TwentyFirst Century: A Perspective, Proceedings of IEEE, vol. 85, N. 10, Oct. 1997, pp. 1622-1638.

[6] K.Biesecker, The Promise of Broadband Wireless, IT Professional, vol. 2, N. 6, Nov.-Dec. 2000, pp.31-39.

[7] G.D. Golden et al, 'Detection Algorithm and Initial Laboratory Results Using V-BLAST Space-Time Communication Architecture', Electronics Letters, vol. 35, No. 1, pp.14-16, $7^{\text {th }}$ January 1999.

[8] On-line: http://www.bell-labs.com/project/blast/

[9] D.R.J. White, "A Handbook Series on Electromagnetic Interference and Compatibility", Don White Consultants Inc., Germantown, Maryland, 1971-1973.

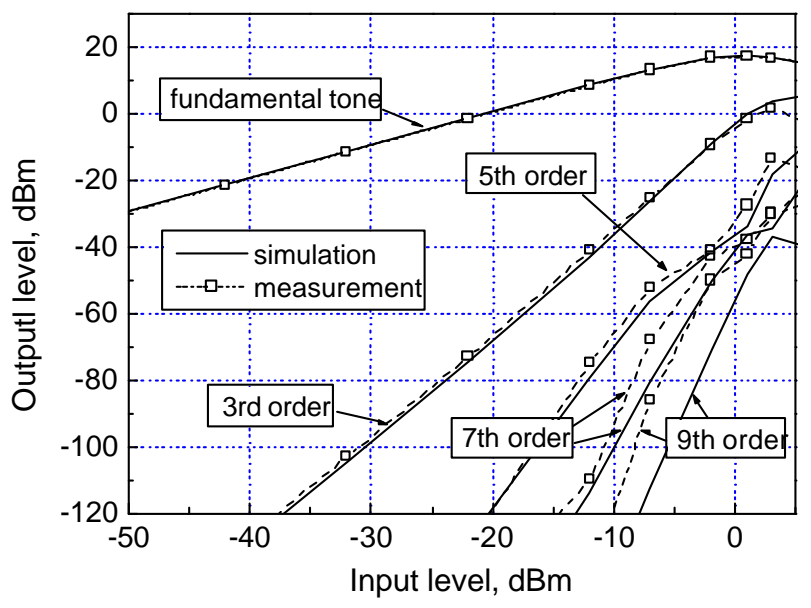

Figure 5. Fundamental and $3^{\text {rd }}$ to $9^{\text {th }}$ harmonics (solid line - simulated, squares - measured) for two-stage MMIC amplifier.

[10]J. Gavan, "Principles of Radio System and Relevant EMI Environmental Effects, Analysis and Computation," in R. Perez (ed.), Handbook on Electromagnetic Compatibility, New York, Academic Press, 1995.

[11]M.C. Jeruchim, P. Balaban, K.S. Shanmugan, Simulation of Communication Systems, Plenum Press: New York, 1992.

[12]J. F. Sevic, M. B. Steer and A. M. Pavio, "Nonlinear analysis methods for the simulation of digital wireless communication systems," International Journal of Microwave and Millimeter-Wave Computer-Aided Engineering, vol. 6, No. 3, 1996, pp.197-216.

[13]S. Loyka, J.R. Mosig, "New behavioral-level simulation technique for $\mathrm{RF} /$ microwave applications. Part I: Basic concepts", Int. Journal of RF and Microwave Computer-Aided Engineering, vol. 10, no. 4, July 2000, pp. 221-237.

[14]S. Loyka, J.R. Mosig, "New behavioral-level simulation technique for RF/microwave applications. Part II: Approximation of nonlinear transfer functions", Int. Journal of RF and Microwave Computer-Aided Engineering, vol. 10, no. 4, July 2000, pp. 238-252.

[15]J. Staudinger, "Applying the Quadrature Modeling Technique to Wireless Power Amplifiers," Microwave Journal, vol. 40, No. 11, Nov. 1997, pp.66-86.

[16]V.I. Mordachev "Express analysis of electromagnetic compatibility of radio electronic equipment with the use of the discrete models of interference and Fast Fourier Transform", Proc. of IX Inter. Wroclaw Symp. on EMC, Poland, Wroclaw, 1988, Part 2, pp.565-570.

[17]S. L. Loyka and V. I. Mordachev, "Identification of Nonlinear Interference Sources with the Use of the Discrete Technique," IEEE EMC Symp., Denver, Colorado, Aug. 24 28, 1998, pp.882-887.

[18]S.L. Loyka, Nonlinear EMI Simulation of an AMDetector at the System Level, IEEE Trans. on EMC, vol. 42, N. 1, Feb. 2000, pp. 97-102. 